\title{
FLAVOR CONSTRAINTS ON THEORY SPACE
}

\author{
E. H. SIMMONS AND R. S. CHIVUKULA \\ Department of Physics, Boston University, \\ 590 Commonwealth Avenue, \\ Boston, MA 02215, USA \\ E-mail:simmons@bu.edu,sekhar@bu.edu \\ N. EVANS \\ Department of Physics, University of Southampton, \\ Highfield, Southampton, SO17 1BJ, UK \\ E-mail: evans@phys.soton.ac.uk
}

\begin{abstract}
Composite Higgs models based on the chiral symmetries of "theory space" can produce Higgs bosons with masses of order $100 \mathrm{GeV}$ from underlying strong dynamics at scales up to $10 \mathrm{TeV}$ without fine tuning. This talk argues that flavor-violating interactions generically arising from underlying flavor dynamics constrain the Higgs compositeness scale to be $\gtrsim 75 \mathrm{GeV}$, implying that significant fine-tuning is required. Bounds from $\mathrm{CP}$ violation and weak isospin violation are also discussed.
\end{abstract}

\section{Introduction}

The Standard Higgs Model employs a fundamental scalar doublet to break the electroweak symmetry and provide fermion masses. Well-known difficulties, including the hierarchy problem and the triviality problem, imply that the Standard Higgs Model is just a low-energy effective theory.

Suppose that the Higgs field $\phi$ is actually a composite ${ }^{1}$ state arising from underlying strong dynamics at a higher energy scale, $\Lambda$. We can estimate the sizes of operators involving $\phi$ in the low-energy effective theory using dimensional analysis ${ }^{2}$. A theory with light scalar particles in a single symmetry-group representation depends ${ }^{3}$ on two parameters: $\Lambda$, the scale of the underlying physics, and $f$, the analog of $f_{\pi}$ in QCD. Our estimates of the low-energy effects of the underlying physics will depend on $\kappa \equiv \Lambda / f$.

Regardless of the precise nature of the underlying strongly-interacting physics that produces $\phi$, there must be flavor dynamics at a scale $\gtrsim \Lambda$ that gives rise to the different Yukawa couplings of the Higgs boson to 
ordinary fermions. If this flavor dynamics arises from gauge interactions it will generally cause flavor-changing neutral currents (as in ETC models ${ }^{4}$ ). Similarly, there are likely to be couplings that violate $\mathrm{CP}$ and weak isospin. This talk reviews the constraints ${ }^{5}$ which FCNC, CP-violation, and weakisospin violation place on composite Higgs models and applies the limits to models developed ${ }^{6,7}$ under the rubric of "theory space" 8 .

\section{Composite Higgs Phenomenology}

\subsection{Flavor}

Quark Yukawa couplings arise from flavor physics coupling the left-handed doublets $\psi_{L}$ and right-handed singlets $q_{R}$ to the strongly-interacting constituents of the composite Higgs doublet. If these new flavor interactions are gauge interactions with gauge coupling $g$ and gauge boson mass $M$, dimensional analysis ${ }^{2}$ estimates the resulting Yukawa coupling is ${ }^{1}$ of or$\operatorname{der} \frac{g^{2}}{M^{2}} \frac{\Lambda^{2}}{\kappa}$. To produce a quark mass $m_{q}$, the Yukawa coupling must equal $\sqrt{2} m_{q} / v$ where $v \approx 246 \mathrm{GeV}$. This implies ${ }^{14}$

$$
\Lambda \gtrsim \frac{M}{g} \sqrt{\sqrt{2} \kappa \frac{m_{q}}{v}} .
$$

If experiment sets a lower limit on $M / g$, eqn.(1) gives a lower bound on $\Lambda$.

Consider the interactions responsible for the $c$-quark mass. Through Cabibbo mixing, these interactions must couple to the $u$-quark as well:

$$
\begin{aligned}
L_{e f f}= & -\left(\cos \theta_{L}^{c} \sin \theta_{L}^{c}\right)^{2} \frac{g^{2}}{M^{2}}\left(\bar{c}_{L} \gamma^{\mu} u_{L}\right)\left(\bar{c}_{L} \gamma_{\mu} u_{L}\right) \\
& -\left(\cos \theta_{R}^{c} \sin \theta_{R}^{c}\right)^{2} \frac{g^{2}}{M^{2}}\left(\bar{c}_{R} \gamma^{\mu} u_{R}\right)\left(\bar{c}_{R} \gamma_{\mu} u_{R}\right) \\
& -2 \cos \theta_{L}^{c} \sin \theta_{L}^{c} \cos \theta_{R}^{c} \sin \theta_{R}^{c} \frac{g^{2}}{M^{2}}\left(\bar{c}_{L} \gamma^{\mu} u_{L}\right)\left(\bar{c}_{R} \gamma_{\mu} u_{R}\right),
\end{aligned}
$$

where $g$ and $M$ are of the same order as those which produce the $c$-quark Yukawa coupling, and $\theta_{L}^{c}, \theta_{R}^{c}$ relate the gauge and mass eigenstates.

The color-singlet products of currents in eqn. (2) contribute to $D$-meson mixing. The left-handed or right-handed current-current operators yield ${ }^{14}$

$$
\left(\frac{M}{g}\right)_{\mathrm{LL}, \mathrm{RR}} \gtrsim f_{D}\left(\frac{2 m_{D} B_{D}}{3 \Delta m_{D}}\right)^{1 / 2} \cos \theta_{L, R}^{c} \sin \theta_{L, R}^{c} \approx 225 \mathrm{TeV}
$$


where $\Delta m_{D} \lesssim 4.6 \times 10^{-11} \mathrm{MeV}^{10}$, and $f_{D} \sqrt{B_{D}}=0.2 \mathrm{GeV}^{11}, \theta_{L, R}^{c} \approx \theta_{C}$. A bound ${ }^{5}$ on the scale of the underlying dynamics follows from eqn. (1):

$$
\Lambda \gtrsim 21 \mathrm{TeV} \sqrt{\kappa\left(\frac{m_{c}}{1.5 \mathrm{GeV}}\right)},
$$

so that $\Lambda \gtrsim 74 \mathrm{TeV}$ for $\kappa \approx 4 \pi$. The LR product of color-singlet currents gives a weaker bound than eqn. (4). The LR product of color-octet currents,

$$
L_{e f f}=-2 \cos \theta_{L}^{c} \sin \theta_{L}^{c} \cos \theta_{R}^{c} \sin \theta_{R}^{c} \frac{g^{2}}{M^{2}}\left(\bar{c}_{L} \gamma^{\mu} T^{a} u_{L}\right)\left(\bar{c}_{R} \gamma_{\mu} T^{a} u_{R}\right),
$$

where $T^{a}$ are the generators of $S U(3)_{C}$, gives a stronger bound ${ }^{5}$ :

$$
\Lambda \gtrsim 53 \mathrm{TeV} \sqrt{\kappa\left(\frac{1.5 \mathrm{GeV}}{m_{c}}\right)} .
$$

Analogous bounds on $\Lambda$ can be derived from neutral Kaon mixing. However, because $m_{s} \ll m_{c}$, while the $d-s$ and $u-c$ mixings are expected to be of comparable size, these bounds on $\Lambda$ are weaker than (4) ${ }^{9}$.

\subsection{Isospin}

Weak-isospin violation is a key issue in composite Higgs models ${ }^{9,13,14,15}$. The standard one-doublet Higgs model has an accidental custodial isospin symmetry ${ }^{16}$, which implies $\rho \approx 1$. While all operators of dimension $\leq 4$ automatically respect custodial symmetry, terms of higher dimension that arise from the underlying physics at scale $\Lambda$ in general will not.

The leading custodial-symmetry violating operator

$$
\frac{\kappa^{2}}{\Lambda^{2}}\left(\phi^{\dagger} D^{\mu} \phi\right)\left(\phi^{\dagger} D_{\mu} \phi\right)
$$

gives rise to a contribution to the $\rho$ parameter ${ }^{13}$

$$
\Delta \rho *=-O\left(\kappa^{2} \frac{v^{2}}{\Lambda^{2}}\right) .
$$

The limit $|\Delta \rho *| \lesssim 0.4 \%$ implies $\Lambda \gtrsim 4 \mathrm{TeV} \cdot \kappa$.

\subsection{CP Violation}

In the absence of additional superweak interactions to give rise to CPviolation in $K$-mixing $(\varepsilon)$, the flavor interactions responsible for the $s$-quark Yukawa couplings must do so. This yields strong bounds on $\Lambda$. Recalling

$$
\operatorname{Re} \varepsilon \approx \frac{\operatorname{ImM}_{12}}{2 \Delta M} \lesssim 1.65 \times 10^{-3},
$$


and assuming that there are phases of order 1 in the $\Delta S=2$ operators analogous to those shown in eqn. (2), we find

$$
\Lambda \gtrsim 120 \mathrm{TeV} \sqrt{\kappa\left(\frac{m_{s}}{200 \mathrm{MeV}}\right)} .
$$

\section{Composite Higgs Bosons from Theory Space}

A set of "theory space" composite Higgs models ${ }^{6,7}$ can be represented as an $N \times N$ toroidal lattice of "sites" connected by "links", using "moose" or "quiver" notation ${ }^{12}$. Each site except $(1,1)$ represents a gauged $S U(3)$ group, while the links represent non-linear sigma fields transforming as $(N, \bar{N})$ 's under the adjacent groups. At the site $(1,1)$, only the $S U(2) \times U(1)$ subgroup of an $S U(3)$ global symmetry is gauged. For simplicity, we will assume the gauge couplings of the $S U(3)$ gauge groups are the same for every site (except $(1,1)$ ). Calling the "pion decay constant" of the chiralsymmetry-breaking dynamics $f$, dimensional analysis ${ }^{2}$ then implies that the scale $\Lambda$ of the underlying high-energy dynamics which gives rise to this theory is $\lesssim 4 \pi f$.

The $2 N^{2}$ Goldstone bosons of the chiral symmetry breaking dynamics are incorporated into the sigma-model fields. As described in ${ }^{6,7}, N^{2}-1$ sets of Goldstone bosons are eaten, $N^{2}-1$ get mass from "plaquette operators" which explicitly break the chiral symmetries, and two sets which are uniform in the ' $\mathrm{u}$ ' or ' $\mathrm{v}$ ' directions, along the lattice axes, remain massless in the very low-energy theory: Both the $\pi_{u}$ and $\pi_{v}$ fields contain $S U(2) \times U(1)$ doublet scalars $\phi_{u}$ and $\phi_{v}$ with the quantum numbers of the Higgs boson. A negative mass-squared for one or both Higgs bosons may be introduced either through a symmetry-breaking plaquette operator at the site $(1,1)^{6}$ or through the effect of coupling the Higgs bosons to the top-quark ${ }^{7}$. In either case, the resulting mass-squared of the Higgs is $\left|m_{h}\right|^{2} \simeq \frac{\lambda v^{2}}{N^{2}}$.

\section{Constraints on Theory Space}

\subsection{Flavor and $C P$}

Because the light quarks and leptons transform under the $S U(2) \times U(1)$ gauge interactions at a site in theory space ${ }^{6,7}$, Yukawa couplings of these fermions to the composite Higgs bosons are generated. The FCNC and CPviolation limits derived in Section 2 therefore apply. Because the composite Higgs bosons are delocalized over the $N^{2}$ sites of theory space, the lower 
bound on $\Lambda$ is a factor of $\sqrt{N}$ stronger. From D-meson mixing, we have

$$
\Lambda \gtrsim 21 \mathrm{TeV} \sqrt{\kappa N\left(\frac{m_{c}}{1.5 \mathrm{GeV}}\right)},
$$

so that $\Lambda \gtrsim \sqrt{N} \cdot 74 \mathrm{TeV}$ for $\kappa=4 \pi$. From CP-violation $(\epsilon)$, we have

$$
\Lambda \gtrsim 120 \mathrm{TeV} \sqrt{\kappa N\left(\frac{m_{s}}{200 \mathrm{MeV}}\right)},
$$

meaning $\Lambda \gtrsim \sqrt{N} \cdot 425 \mathrm{TeV}$ for $\kappa=4 \pi$.

A significant advantage of theory space models is supposed to be their ability to produce a light Higgs without fine-tuning. We must check how compatable this is with the FCNC and CP-violation constraints above.

The most important corrections to the Higgs boson masses arise from the interactions added to give rise to the top-quark mass. The fermion loop Coleman-Weinberg ${ }^{17}$ contribution to the Higgs mass-squared is of order

$$
\left|\delta m_{H}^{2}\right| \simeq \frac{N_{c} y_{t}^{2} M^{2}}{16 \pi^{2}} \approx \frac{N_{c} y_{t}^{4}}{\left(16 \pi^{2}\right)^{2}} \Lambda^{2},
$$

where $N_{c}=3$ accounts for color. In this case, the absence of fine-tuning $\left(\delta m_{H}^{2} / m_{H}^{2} \lesssim 1\right)$ implies

$$
\Lambda \lesssim \frac{16 \pi^{2} \sqrt{\lambda} v}{\sqrt{N_{c}} y_{t}^{2} N} \approx \frac{22 \mathrm{TeV} \sqrt{\lambda}}{N} .
$$

Comparing eqs. (14) and (11) we see that remaining consistent with the low-energy constraints makes fine-tuning inevitable for large $N$. Even for the smallest $N$, some fine-tuning will be required. For example, for $N=2(N=\sqrt{2})$ fine-tuning on the order of $1 \%(3 \%)$ is required by the bound on D-meson mixing. If the bound from CP violation (10) must also be satisfied, the fine-tuning required is of order .04\% (0.09\%).

\section{Weak Isospin Violation}

The kinetic energy terms for the light composite Higgses include isospinviolating interactions ${ }^{5}$

$L_{k i n} \supset-\frac{1}{6 N f^{2}}\left[\left(\partial_{\mu} \phi_{u}^{\dagger} \phi_{u}\right)^{2}-\left(\partial_{\mu} \phi_{u}^{\dagger} \phi_{u}\right)\left(\phi_{u}^{\dagger} \partial^{\mu} \phi_{u}\right)+\left(\phi_{u}^{\dagger} \partial^{\mu} \phi_{u}\right)^{2}\right]+u \leftrightarrow v$.

The resulting contribution to the $\rho$ parameter is ${ }^{5}$

$$
\Delta \rho^{\star}=\alpha \Delta T=\frac{v^{2}}{4 N^{2} f^{2}}\left(1-\frac{\sin ^{2} 2 \beta}{2}\right) .
$$


Current limits derived from precision electroweak observables ${ }^{15}$ require that $\Delta T \lesssim 0.5$ at $95 \%$ confidence level for a Higgs mass less than $500 \mathrm{GeV}$. The bound in eqn. 16 implies that

$$
\Lambda \simeq 4 \pi f \gtrsim \frac{25 \mathrm{TeV}}{N}\left(1-\frac{\sin ^{2} 2 \beta}{2}\right)^{1 / 2} .
$$

Comparison with eq.(14) shows that the underlying strong dynamics cannot be at energies $\ll 10 \mathrm{TeV}$, even if the high-energy theory contains approximate flavor and $\mathrm{CP}$ symmetries that avoid the limits of eqs. $(4,10)$.

\section{Discussion}

Theory space models propose to provide a naturally light composite Higgs boson without relying on approximate symmetries of the high-energy underlying strong dynamics. This talk argues that the low-energy structure of composite Higgs models does not automatically make them invulnerable to constraints from FCNC, CP-violation, or weak-isospin violation. Assumptions about symmetries of the underlying dynamics are required (see, e.g., discussion in ref. ${ }^{5}$ ).

For theory space models based on an $N \times N$ toroidal lattice ${ }^{6,7,19,18}$, the lower limit from FCNC on the scale of strong dynamics is $\Lambda \geq 74 \mathrm{TeV} \sqrt{N}$, implying a minimum bound of $105 \mathrm{TeV}$. However, if fine-tuning of the higgs mass is to be avoided in such models, $\Lambda \leq 22 \mathrm{TeV} \sqrt{\lambda} / N$; preventing FCNC then leads to fine-tuning at the level of $10 / N^{3} \%$. The lower limits on $\Lambda$ from weak isospin violation are weaker than those from FCNC (but hard to avoid), while those from $\mathrm{CP}$-violation can be much stronger.

\section{Acknowledgments}

Supported in part by Department of Energy under DE-FG02-91ER40676 and by National Science Foundation grant PHY-0074274.

\section{References}

1. D. B. Kaplan and H. Georgi, Phys. Lett. B136 (1984) 183; Phys. Lett. B145 (1984) 216; T. Banks, Nucl. Phys. B 243 (1984) 123; D. B. Kaplan, H. Georgi, and S. Dimopoulos, Phys. Lett. B136 (1984) 187; M. J. Dugan, H. Georgi, and D. B. Kaplan, Nucl. Phys. B 254 (1985) 299.

2. A. Manohar and H. Georgi, Nucl. Phys. B234 (1984) 189; H. Georgi and L. Randall, Nucl. Phys. B276 (1986) 241; T. Appelquist, Scottish Summer School (1980) 385. 
3. H. Georgi, Phys. Lett. B298 (1993) 187, hep-ph/9207278.

4. S. Dimopoulos and L. Susskind, Nucl. Phys. B155 (1979) 237; E. Eichten and K. Lane, Phys. Lett. B90 (1980) 125.

5. R. S. Chivukula, N. Evans and E. H. Simmons, Phys. Rev. D 66 (2002) 035008 [arXiv:hep-ph/0204193].

6. N. Arkani-Hamed, A. G. Cohen and H. Georgi, Phys. Lett. B 513 (2001) 232 [arXiv:hep-ph/0105239].

7. N. Arkani-Hamed, A. G. Cohen, T. Gregoire and J. G. Wacker, JHEP 0208 (2002) 020 [arXiv:hep-ph/0202089].

8. N. Arkani-Hamed, A. G. Cohen and H. Georgi, Phys. Rev. Lett. 86 (2001) 4757 [arXiv:hep-th/0104005].

9. R. S. Chivukula, B. A. Dobrescu and E. H. Simmons, Phys. Lett. B 401 (1997) 74 [arXiv:hep-ph/9702416].

10. D.E. Groom et al, The European Physical Journal C15 (2000) 1, and 2001 off-year partial update for the 2002 edition available on the PDG WWW pages (URL: http://pdg.lbl.gov/)

11. See, for example, A. Ali Khan et al. [CP-PACS Collaboration], Phys. Rev. D 64, 034505 (2001) [arXiv:hep-lat/0010009].

12. H. Georgi, Nucl. Phys. B 266, 274 (1986); M. R. Douglas and G. W. Moore, arXiv:hep-th/9603167.

13. R. S. Chivukula and E. H. Simmons, Phys. Lett. B 388 (1996) 788 [arXiv:hep$\mathrm{ph} / 9608320]$.

14. R. S. Chivukula, E. H. Simmons and B. A. Dobrescu, arXiv:hep-ph/9703206.

15. R. S. Chivukula and N. Evans, Phys. Lett. B 464 (1999) 244 [arXiv:hepph/9907414]. ; R. S. Chivukula, C. Hoelbling and N. Evans, Phys. Rev. Lett. 85 (2000) 511 [arXiv:hep-ph/0002022]. ; R. S. Chivukula, arXiv:hep-ph/0005168. ; R. S. Chivukula and C. Hoelbling, in Proc. of the APS/DPF/DPB Summer Study on the Future of Particle Physics (Snowmass 2001) ed. R. Davidson and C. Quigg, arXiv:hep-ph/0110214.

16. S. Weinberg, Phys. Rev. D19 (1979) 1277; L. Susskind, Phys. Rev. D20 (1979) 2619; P. Sikivie, et. al., Nucl. Phys. B173 (1980) 189.

17. S. R. Coleman and E. Weinberg, Phys. Rev. D 7 (1973) 1888.

18. N. Arkani-Hamed, A. G. Cohen, E. Katz and A. E. Nelson, JHEP 0207 (2002) 034 [arXiv:hep-ph/0206021].

19. N. Arkani-Hamed, A. G. Cohen, E. Katz, A. E. Nelson, T. Gregoire and J. G. Wacker, JHEP 0208 (2002) 021 [arXiv:hep-ph/0206020]. 